\title{
Diode Laser Biostimulatory Effect on Dental Implant Inserted into Narrows Mandibular Ridges: Clinico-Radiographic Evaluation
}

\section{Hamdy A Abol-Khair ${ }^{1}$, Mostafa M Hosny ${ }^{2}$, Hisham A Abozeid ${ }^{3}$ and Abdel-Fattah M Amer ${ }^{4 *}$}

${ }^{1}$ Assistant Lecturer, Department of Oral Medicine, Periodontology, Diagnosis and Oral Radiology Faculty of Dentistry, Al-Azhar University, Cairo, Egypt

${ }^{2}$ Assistant Professor, Department of Oral Medicine, Periodontology, Diagnosis and Oral Radiology, Faculty of Dental Medicine Al-Azhar University, Cairo, Egypt

${ }^{3}$ Lecturer, Department of Oral Medicine, Periodontology, Diagnosis and Oral

Radiology Faculty of Dentistry, Al-Azhar University, Cairo, Egypt

${ }^{4}$ Professor of Oral Medicine, Periodontology, Diagnosis and Oral Radiology, Faculty of

Dental Medicine Al-Azhar University, Cairo, Egypt

*Corresponding Author: Abdel-Fattah M Amer, Professor of Oral Medicine, Periodontology, Diagnosis and Oral Radiology, Faculty of Dental Medicine Al-Azhar University, Cairo, Egypt.
Received: August 27, 2021

Published: September 27, 2021

(C) All rights are reserved by Abdel-Fattah $\mathbf{M}$

Amer., et al.

\section{Abstract}

Purpose: To evaluate possible effect of simultaneous Laser therapy application on dental implant inserted into narrow alveolar ridges after crestal splitting using a piezoelectric device.

Subjects and Methods: Thirty-two dental implants were inserted into 16 patients exhibiting partially edentulous mandibular posterior sites, applying split mouth technique to classify them into two groups: Group 1: received split-crest technique by ultrasonic bone surgery with implant placement and PRF only. Group 2: received split-crest technique by ultrasonic bone surgery with the implant. Placement and PRF + biostimulation of implant sites by diode laser. Clinical evaluation was carried out using: site-specific Gingival Index (GI), Plaque Index (PII) and Pocket depth (PD). These were recorded immediately after implant loading, at one, three, and six months later. Implant stability was evaluated by resonance frequency analysis (RFA) at the baseline and immediately before implant loading. Radiographic evaluation was carried out preoperative using (CBCT) to assess the bone height and thickness (to guide the implant treatment plan), immediately after surgery at time of loading, as well as 6 months after loading to evaluate the ridge width, crestal bone loss, and bone density.

Results: There were no statistically significant differences regarding GI, PII, and PD measured in the two groups after one as well as three months. After 6 months; Group I showed a statistically significantly lower scores than Group II, for GI the P-value $=0.021$, Effect size $=1.416$ ), for PII the P-value $=0.027$, Effect size = 1.333), and for PD the P-value $=0.008$, Effect size $=0.386$ ). Radiographically, there was no statistically significant difference between median crestal bone height measurements in the two groups immediately after loading as well as after six months. The two groups showed a statistically significant increase in crestal bone height measurements denoting crestal bone loss (P-value $<0.001$, Effect size $=3.701$ ) and (P-value $=0.001$, Effect size $=3.265$ ), respectively. No statistically significant difference between the two groups in ridge width, bone density, and Implant stability evaluated RFA.

Keywords: Laser Biostimulation; PRF; Piezoelectric Surgery; Ridge Splitting; Implant Stability; CBCT 


\section{Introduction}

Dento-alveolar ridges that are narrow and atrophic provide a significant obstacle for dental implant insertion [1,2], as appropriate amount of alveolar bone is necessary around implant after its placement. Thickness of vestibular and buccal surrounding bone lamellae should be around $1 \mathrm{~mm}$ in the horizontal dimension [3]. According to Wolff's law, "bone remodels in proportion to the forces applied". When a bone's function is altered (teeth loss), its internal architecture and external configuration are altered as well [4]. Misch mentioned that, teeth absence result in lack of stimulation to the alveolar bone, which decrease in trabeculae and bone density, as well as a loss of width and height; degradation of edentulous alveolar ridges is enhanced by inflammatory local processes [5].

The presence of narrow alveolar ridges is a challenge in field of dental implants. Hence several attempts and efforts were directed toward the expansion of these ridges. In this respect, to obtain a horizontal bone expansion, a variety of procedures as ridge splitting, guided bone regeneration, and bone block grafting may be used. Ridge-splitting is a faster way to predictably extend and graft an atrophic ridge with no need for a second surgical site harvesting $[1,6,7]$. The split-crest technique is a flexible technique that allows many modifications, which are can performed with a choice of devices for bone cutting. The most common instruments implemented were fissure burs [8], osteotomy micro-saw/discs [9-11], chisels [4,12-14] and piezo-electric knives [10,14-17]. The piezo surgery has several advantages including: clear vision of the surgical area from the pressurized irrigation and cavitation effect; hemostasis is ensured through the cavitation effect; bone sectioning can be performed with micrometric sensitivity; avoiding the risk of damage to adjacent soft tissue while cutting; healing occurs fast because no damage is inflicted on the living bone morphogenetic protein release as well as ease of harvesting intra- or extra-oral autogenous graft. Its ability to be inserted with various angles makes it easily used in areas where it is difficult to see and reach. Due to absence of macro-vibrations, patients feel very comfortable during surgeries under local anesthesia [18].

Low-level laser therapy (LLLT) is a well-accepted adjuvant medical method for improving wound healing processes in general and treating functional impairments [10]. A great number of researches on its use in periodontal therapy have been carried out $[11,13]$. The term "phototherapy" refers to therapeutic procedures that use lasers or incoherent light at low or high energy levels. Laser therapy, in particular, has been increasingly adopted and successfully employed clinically in dentistry since the early 1990s. Lasers have a variety of tissue interactions, such as ablation or vaporization, hemostasis, microbial suppression and destruction, and biological impacts, such as bio-stimulation (photo-bio-modulation), which cause a variety of favorable therapeutic effects and biological responses. Thus, the use of lasers is thought to be useful and appropriate for treating several inflammatory and infectious disorders including periodontal disease $[14,16]$. Laser therapy may reduce a patient's physical and mental stress, as well as intra- and postoperative pain [17].

One of new aspect is to use Laser as an adjuvant therapy to promote a potential effect for bone regeneration, through its impact on ATP generation, reactive oxygen species, and transcription factors; as Laser treatment influences cell proliferation and migration [19]. This mechanism increases the likelihood of bone regeneration and callus development $[20,21]$. In this respect, a study found that Laser therapy can improve bone density while also promoting anti-inflammatory and analgesic effects in maxillofacial bony abnormalities [22]. Studies have found that the use of LLL improves bone growth [23], as distraction osteogenesis using LLL resulted in greater bone mineral density of freshly produced bone. Another study [27] reported that LLL was successfully used in a block allograft for bone defect regeneration. Wound healing is divided into three stages: the inflammatory phase, the cell proliferation phase, and tissue remodeling phase.

Laser bio-stimulation largely influences the cell proliferation phase of the wound healing process [24]. Mitochondria are sensitive to monochromatic near-infrared light, and laser light appears to boost respiratory metabolism in some cells $[25,26]$. Laser light treatment influences processes as fibroblast proliferation, procollagen, and collagen synthesis, growth factor production (including transforming growth factor [TGF], keratinocyte growth factor $[\mathrm{KGF}]$ and platelet-derived growth factor [PDGF]), macrophage stimulation, lymphocyte stimulation [27] and an increase in the rate of extracellular matrix production [28].

Platelet rich fibrin (PRF) has a very significant slow continuous release of growth factors for at least 1 week and up to 28 days $[29,30]$. PRF could inspire its environment for an extended period of time during wound healing [31]. PRF has been shown to play 
an important function in bone healing and quality improvement in studies [15,32]. It has been claimed that PRF able to improve the stability of dental implants by influencing the development and proliferation of osteoblasts [33]. PRF is a second generation platelet concentrate made up of viable platelets that release a variety of growth factors including platelet derived growth factor, vascular endothelial growth factor, transforming growth factor, insulin-like growth factor, epidermal growth factor, and basic fibroblast growth factor. PRF clot's robust fibrin matrix provides a scaffold for transporting cells that are needed for tissue regeneration while also protecting growth factors from proteolysis, and it has the advantage of being entirely autologous and cost-effective $[9,12]$.

In view of these it was hypothesized that application of diode laser immediately to dental implants inserted into narrow mandibular alveolar ridges that expanded by means of piezo surgery may have added value through initiation of bone regeneration effect. The present study was designed and performed to test this hypothesis through recording of clinical parameters as well as through radiographic examination.

\section{Subjects and Methods}

This study was designed as a randomized clinical trial. Patients with narrow ridges seeking implant placement were incorporated. They were 16 subjects; nine females (56.3\%) and seven males (43.7\%); mean age were $42.7+10.5$ years old; were selected from Outpatient Clinic of the Department of Oral Medicine, Periodontology, Oral Diagnosis and Oral Radiology, Faculty of Dental Medicine, Boys, Cairo, Al- Azhar University.

\section{Eligibility criteria of population:}

- Inclusion criteria: Adults exhibiting initial insufficiently bone ridge ranged from ( $3-5 \mathrm{~mm}$ ) with no vertical bone defect; exhibiting good oral hygiene; and are free from systemic diseases that may influence the outcome of the therapy.

- Exclusion criteria: Heavy smokers; patient treated by immunosuppressive chemotherapy or radio therapy, and patients allergic to any material or medication used in the study.

- Ethical consideration: Nature of the study was explained to patients and they signed written consent forms.
Sample size calculation

This was carried out on the basis of previous work [23], thus a sample size of 16 implants in each group has an $80 \%$ power to detect a difference between means of 10.26 with a significance level (alpha) of 0.05 (two-tailed) and 95\% confidence intervals. In $80 \%$ (the power) of those experiments, $\mathrm{P}$ value will be less than 0.05 (two-tailed); so the results will be deemed "statistically significant". In the remaining $20 \%$ of the experiments, the difference between means will be deemed "not statistically significant". Report created by GraphPad StatMate 2.00:

$N=\left(\frac{Z \delta}{M E}\right)^{2}$

Where; $(\mathrm{Z})$ is the $\mathrm{Z}$ score, $(\delta)$ is the standard deviation and (ME) margin of error

$M E=Z \cdot \frac{\delta}{\sqrt{n}}$

Confidence level 1 90\% 95\% 98\% 99\%

Z score value 1.641 .962 .332 .575

Intervention

Split mouth technique will be used and every patient will receive two types of treatment modalities in both sites of bilateral edentulous area: Group 1: received split-crest technique by ultrasonic bone surgery with implant placement and PRF only. (Nucleoss implant system ${ }^{\mathrm{TM}}$ T6 line Menderes, Izmir -Turkey). Group 2: received split-crest technique by ultrasonic bone surgery with implant placement and PRF + bio-stimulation of implant sites by diode laser. (Nucleoss implant system ${ }^{\mathrm{TM}}$ T6 line Menderes, Izmir Turkey). Each implant was loaded according to implant stability evaluated by resonance frequency analysis. Prior surgery, patients were instructed with proper oral hygiene measures. Pre-surgical mouth rinse was carried out with $0.12 \%$ chlorhexidine digluconate rinse, while povidone iodine solution was used to perform the extra oral antisepsis. At least two hours prior to surgery, 1g of amoxicillin was administered to all patients. Antibiotic therapy was continued for one week postoperatively, and under local anesthesia surgical procedure was done according to the proposed technique.

\section{Ridge splitting (RS) group with (PRF only)}

Under local anesthesia, a papillary sparing crestal incision was performed on the atrophic ridge; incision was followed by two 
vertical releasing incisions beyond the mucogingival line. Then a full thickness mucoperiosteal flap was raised, and when the bone surface was exposed the planned osteotomies were outlined, using tip number one at low power, in order to avoid oscillation of the tip and obtain a cut depth $1 \mathrm{~mm}$. The first osteotomy was carried out at the center of the occlusal aspect of the ridge, extending the incision in anteroposterior direction for the planned length, subsequently, the osteotomy lines were traced using the tips progressively in order of size, varying the power level of the characteristics of the incision change too. In this way once the osteotomy lines have been outlined, the tips were used in progression from number one to number five to deepen the osteotomies (Figure 1).

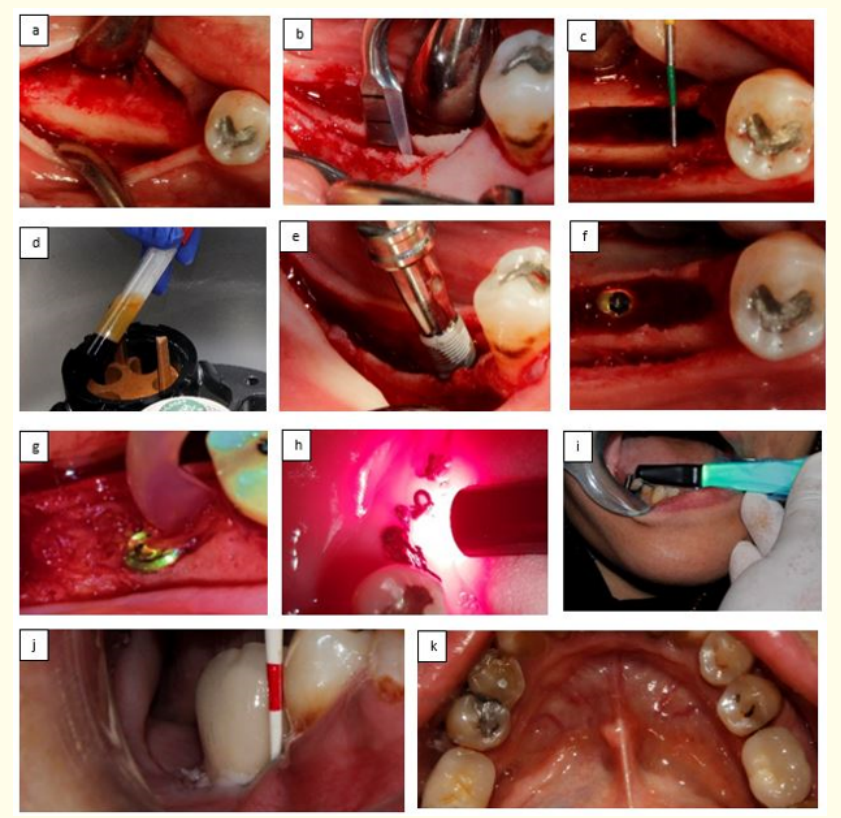

Figure 1: (a) Flap reflection, (b) ridge splitting using piezotome, (c) gained space between buccal and lingual plates of alveolar bone, (d) PRF preparation, (e) implant insertion, (f) implant fully engaged between bone plates, (g) application of PRF around implant in the created space, (h) diode laser application post-operatively, (i) measuring implant stability using Osstell Beacon, (j) probing depth around loaded implant and (k) bilaterally loaded implants.

\section{PRF preparation}

An amount of $5 \mathrm{ml}$ whole venous blood was collected in each of two sterile vacutainer tubes of $6 \mathrm{ml}$ capacity without anticoagulant. The tubes were then placed in a centrifugal machine at 3000 revolutions per minute (rpm) for 10 minutes, after which they were settled into the following layers: red lower fraction containing red blood cells, upper straw colored cellular plasma and the middle fraction containing the fibrin clot. The upper straw colored layer is then removed and middle fraction is collected, $2 \mathrm{~mm}$ below lower dividing line, which is the PRF.

The mechanism which is followed here is that, fibrinogen which is initially concentrated in the high part of the tube, combines with the circulating thrombin due to centrifugation, to form fibrin. A fibrin clot is then obtained in the middle of the tube, just between the red corpuscles at the bottom and acellular plasma at top. Platelets are trapped massively in the fibrin meshes. Quick handling is the only way to obtain a clinically usable PRF clot. PRF protocol makes it possible to collect a fibrin clot charged with serum and platelets. By driving out the fluids trapped in the fibrin matrix, practitioners can obtain very resistant autologous fibrin membranes.

\section{Follow up observation}

Clinical parameter including: i. Site-specific Gingival Index was evaluated after loading by 1, 3 and 6 months [19]. ii. Implant stability was evaluated by resonance frequency analysis (RFA) [20] at base line and immediately before loading. iii. Pocket depth [21] was measured after loading by 1, 3 and 6 months. iv. Plaque index [22] was evaluated after loading at 1, 3 and 6 months.

Radiological assessment: Preoperative Cone beam computerized tomography (CBCT) was taken for assessment of bone height and thickness (implant treatment plan), immediately after surgery, at time of loading and 6 months after loading to evaluate ridge width, crestal bone loss and bone density (Figure 2).

\section{Ethical consideration}

Research protocol was approved by the ethical committee, Faculty of Dental Medicine (Boys, Cairo), Al- Azhar University, Egypt. 


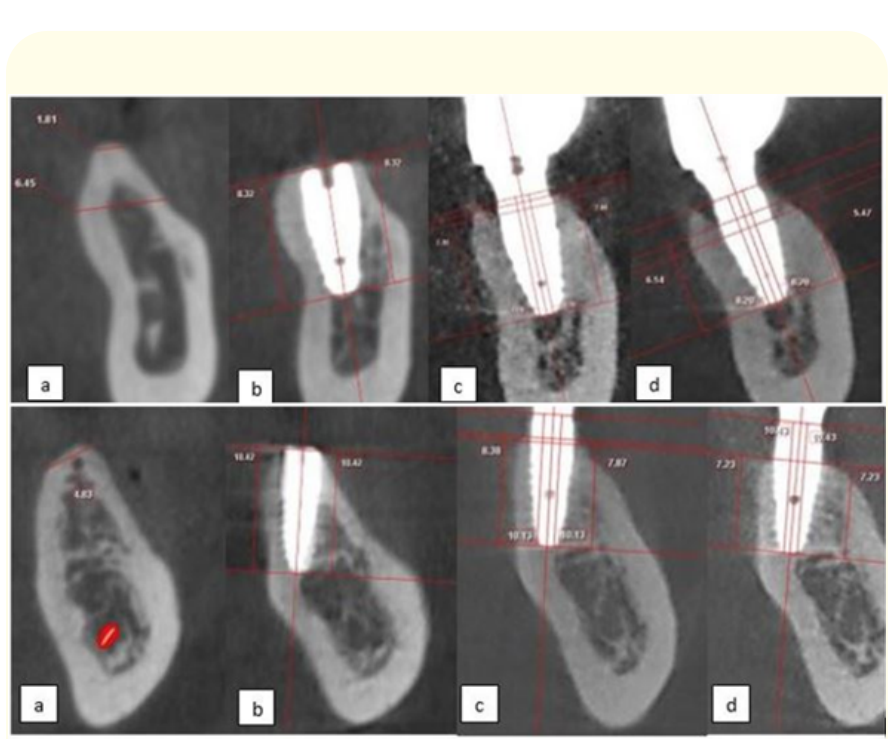

Figure 2: (a) Pre-operative radiograph, (b) Immediately after implant insertion, (c) Immediately after loading, (d) Six months after loading.

\section{Results}

The present study was conducted on 16 subjects; nine females $(56.3 \%)$ and seven males (43.7\%). The mean and standard deviation values for age were 42.7 (10.5) years old.

\section{Clinical parameters}

Gingival index (GI): There was no statistically significant difference between median GI scores in the two groups after one as well as three months. After six months; Group I showed statistically significantly lower median GI score than Group II (P-value = 0.021 , Effect size $=1.416$ ). As regards the changes by time within Group I; there was a statistically significant change (P-value = 0.019 , Effect size $=0.249$ ). Pair-wise comparisons between the time periods revealed that there was a statistically significant decrease in GI scores after three months followed by non-statistically significant change from three to six months. The median GI score after six months showed statistically significantly lower median score compared to one month's score. While for Group II; there was a statistically significant change (P-value $=0.010$, Effect size $=$ 0.289). Pair-wise comparisons between the time periods revealed that there was a statistically significant decrease in GI scores after three months followed by a statistically significant increase from three to six months. The median GI score after six months showed statistically significantly higher median score compared to one month's score (Table 1).

\section{Crestal bone height ( $\mathrm{mm})$}

\section{Crestal bone height measurements}

There was no statistically significant difference between median crestal bone height measurements in the two groups immedi-

\begin{tabular}{|c|c|c|c|c|c|c|}
\hline \multirow{2}{*}{ Time } & \multicolumn{2}{|c|}{ Group I (n= 16 implants) } & \multicolumn{2}{c|}{ Group II (n = 16 implants) } & P-value & Effect size \\
\cline { 2 - 6 } & Mean (SD) & Median (Range) & Mean (SD) & Median (Range) & & (d) \\
\hline 1 month & $0.42(0.21)$ & $0.4(0.1-0.7)^{\mathrm{A}}$ & $0.39(0.21)$ & $0.35(0.2-0.7)^{\mathrm{B}}$ & 0.503 & 0.34 \\
\hline 3 months & $0.2(0.14)$ & $0.2(0-0.5)^{\mathrm{B}}$ & $0.23(0.05)$ & $0.2(0.2-0.3)^{\mathrm{C}}$ & 0.482 & 0.357 \\
\hline 6 months & $0.29(0.2)$ & $0.2(0-0.5)^{\mathrm{B}}$ & $0.51(0.23)$ & $0.6(0.2-0.7)^{\mathrm{A}}$ & $0.021^{*}$ & 1.416 \\
\hline P-value & \multicolumn{2}{|c|}{$0.019^{*}$} & \multicolumn{2}{|c|}{$0.010^{*}$} & & \\
\hline Effect size (w) & \multicolumn{2}{|c|}{0.249} & & \\
\hline
\end{tabular}

Table 1: Descriptive statistics and results of Wilcoxon signed-rank test for comparison between GI scores in the two groups and Friedman's test for comparison between GI scores at different time periods within each group.

*: Significant at $\mathrm{P} \leq 0.05$, Different superscripts in the same column indicate statistically significant change by time.

ately after loading as well as after six months (Figure 3). As regards the changes by time within both groups; there was a statistically significant increase in crestal bone height measurements denoting crestal bone loss (P-value $<0.001$, Effect size $=3.701$ ) and (P-value $=0.001$, Effect size $=3.265$ ), respectively. 


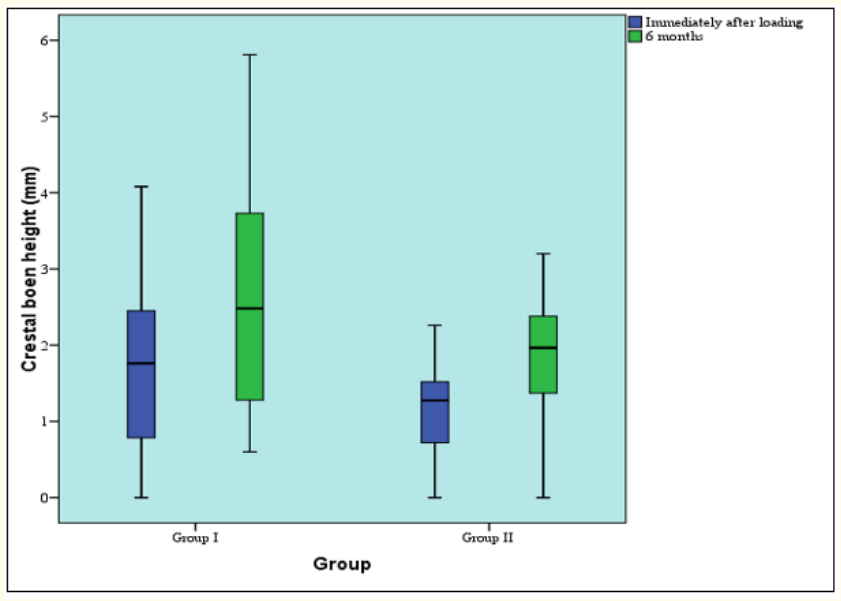

Figure 3: Box plot representing median and range values for crestal bone height measurements in the two groups.

\section{Amount of crestal bone loss (mm)}

Crestal bone loss was calculated as: (Bone height after six months -bone height immediately after loading). There was no statistically significant difference between median amounts of crestal bone loss in the two groups after six months (Table 2).

\begin{tabular}{|c|c|c|c|c|c|}
\hline \multicolumn{2}{|c|}{$\begin{array}{c}\text { Group I (n = 16 } \\
\text { implants) }\end{array}$} & \multicolumn{2}{c|}{$\begin{array}{c}\text { Group II (n= 16 } \\
\text { implants) }\end{array}$} & $\begin{array}{c}\text { P- } \\
\text { value }\end{array}$ & $\begin{array}{c}\text { Effect } \\
\text { size (d) }\end{array}$ \\
\cline { 1 - 4 } $\begin{array}{c}\text { Mean } \\
\text { (SD) }\end{array}$ & $\begin{array}{c}\text { Median } \\
\text { (Range) }\end{array}$ & $\begin{array}{c}\text { Mean } \\
\text { (SD) }\end{array}$ & $\begin{array}{c}\text { Median } \\
\text { (Range) }\end{array}$ & & \\
\cline { 1 - 3 } 0.93 & $0.79(0.18-$ & 0.78 & $0.7(0-2.11)$ & 0.587 & 0.274 \\
$(0.6)$ & $2.57)$ & $(0.54)$ & & & \\
\hline
\end{tabular}

Table 2: Descriptive statistics and results of Wilcoxon signed-rank test for comparison between crestal bone loss ( $\mathrm{mm})$ in the two groups.

$*$ : Significant at $\mathrm{P} \leq 0.05$.

\section{Implant stability}

Implant stability was evaluated by resonance frequency analysis (RFA) [20] at base line and immediately before loading.

Group I recorded ISQ values at baseline ranging between 73 - 81 $($ mean was $78.58+2.17)$ a range between $74-84$ (mean $80.08+$
2.98) and a range between70 -81 (mean $76+3.15$ ). Group II recorded ISQ values at baseline ranging between $73-89$ (mean was $77.25+2.87$ ) a range between $63-80$ (mean $76.00+3.45)$ and a range between 68 - 77 (mean $72.50+3.85$ ). The comparison between the two groups showed statistically significant differences between the two groups (Figure 4 illustrating these recorded data).

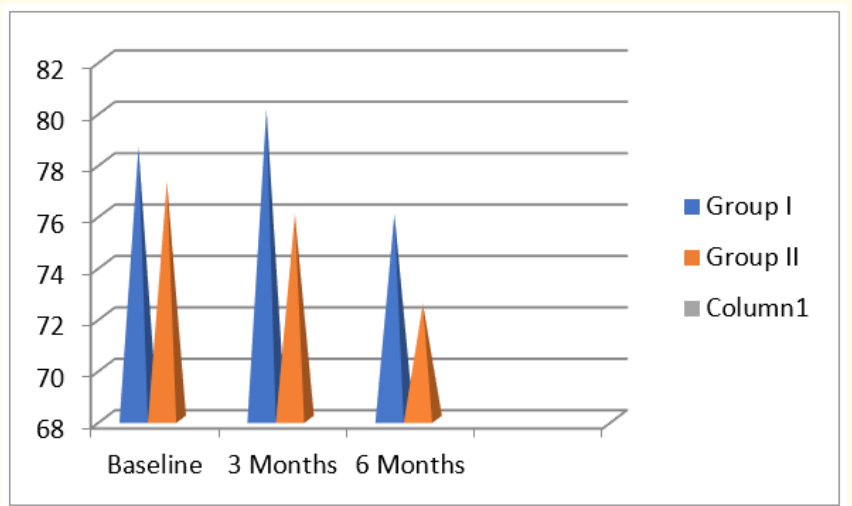

Figure 4: The comparison between the two groups included in the present study regarding the values of ISQ denoting the implant stability recorded at different intervals.

\section{Discussion}

Mester., et al. (1971) [34] published the first report on biological laser stimulation and whether low-level laser therapy accelerates ossification or not. Several studies have found that using a low-level laser after implant implantation promotes osteointegration due to rapid bone turnover. When compared to other laser systems, the diode laser offers superior tissue transmission efficiency; hence this laser was used to stimulate ossification. Low-level laser therapy has been shown to shorten the healing time of bone fractures [35,36]. Following the split-crest operation, an immediate loading implant may exhibit the same biologic activity as a fresh socket implant. In these operations, just half of the implant length is inserted into the native bone with the proper insertion torque, while the remaining coronal piece is only in touch with the bone walls. Similarly, the width of the coronal gap between the implant surface and bone walls at the time of implant placement represents 
a critical point for bone healing in fresh extraction socket implants, because as the gap widened, the amount of bone-to-implant contact decreased and the point of highest bone-to-implant contact shifted apically [37].

Low-intensity laser irradiation has been shown to have a positive effect on preserving crestal bone surrounding implants in implant dentistry. A study [38] revealed that the alveolar bone height was preserved in the side exposed to the low-level laser, and based on the positive results, the researchers concluded that applying the laser to immediately loaded implants; it preserves the supporting alveolar bone and increases bone density when compared to nonlased implants. In the current study, the implants on one side of the same patient were exposed to diode laser irradiation, while the implants on the other side were not exposed to any laser therapy. Both implants were then early loaded and followed up on for 6 months to evaluate the marginal bone around the implants. The results demonstrated that the bone loss surrounding the diode laser-exposed implants was of no differences with the marginal bone loss around the implants on the other side.

The current study's findings are not supporting previous research that found that exposing the area around the implants to low-level laser irradiation could help protect the bone around the implants [38]. Another study [39] reported that when implants on one side of the same patient were exposed to diode laser irradiation while implants on the other side were not, both implants were early loaded and followed up for 6 months to evaluate the marginal bone around the implants.

The results demonstrated that there was no significant variation in peri-implant probing depth between the two sides. this is contrary to the recent study findings which stated that after six months; Group I showed statistically significantly lower mean probing depth (PD) than Group II (P-value $=0.008$, Effect size $=$ 0.386). But there was no statistically significant difference between mean PD measurements in the two groups after one as well as three months. A previous study [39] reported that probing depth measurements were taken from four sites around the implant (buccal, lingual, mesial and distal), while in the present study we measured PD only mesial and distal aspects. Additionally, the previous study found that the bone loss surrounding the diode laser-exposed im- plants was smaller than the marginal bone loss around the implants on the control side. The difference was significant at three and six months. But in recent study there was no statistically significant difference between median amounts of crestal bone loss in the two groups after six months. Putting into consideration that mentioned study measured crestal bone loss mesial and distal to implant by two dimensional radiographs, but in the present study crestal bone loss was measured buccal and lingual to implant using three dimensional radiographs (CBCT).

\section{Conclusion}

Diode laser has a noticable positive biostimulatory effect on both soft and hard tissue around dental implants inserted in challenging narrow mandibular ridges after ridge splitting procedure.

\section{Bibliography}

1. Moro A., et al. "Alveolar ridge split technique using piezosurgery with specially designed tips". BioMed Research International (2017).

2. Pavliková G., et al. "Piezosurgery in oral and maxillofacial surgery". International Journal of Oral and Maxillofacial Surgery 40.5 (2011): 451-457.

3. Nedir R., et al. "A 7-year life table analysis from a prospective study on ITI implants with special emphasis on the use of short implants: Results from a private practice". Clinical Oral Implants Research 15.2 (2004): 150-157.

4. Pelo S., et al. "Augmentation of the atrophic edentulous mandible by a bilateral two-step osteotomy with autogenous bone graft to place osseointegrated dental implants". International Journal of Oral and Maxillofacial Surgery 39.3 (2010): 227234.

5. Misch C. "ARABIC-Contemporary Implant Dentistry". Elsevier Health Sciences (2007).

6. Zakhary I., et al. "Alveolar ridge augmentation for implant fixation: status review". Oral Surgery, Oral Medicine, Oral Pathology, and Oral Radiology 114.5 (2012): S179-S189.

7. Stricker A., et al. "The bone splitting stabilisation technique--a modified approach to prevent bone resorption of the buccal wall". Oral Health and Dental Management 13.3 (2014): 870876. 
8. Han J., et al. "The effects of bone grafting material and a collagen membrane in the ridge splitting technique: an experimental study in dogs". Clinical Oral Implants Research 22.12 (2011): 1391-1398.

9. Ramaprabha G and Jacob TS. "Platelet rich fibrin-A boon for periodontal regeneration". Indian Journal of Multidisciplinary Dentistry 4.2 (2014): 956.

10. Mester E., et al. "The biomedical effects of laser application". Lasers in Surgery and Medicine 5.1 (1985): 31-39.

11. Ishikawa I., et al. "Application of lasers in periodontics: true innovation or myth?" Periodontology 200050.1 (2009): 90-126.

12. Wu C., et al. "Platelet-rich fibrin increases cell attachment, proliferation and collagen-related protein expression of human osteoblasts". Australian Dental Journal 57.2 (2012): 207-212.

13. Schwarz F., et al. "Laser application in non-surgical periodontal therapy: a systematic review". Journal of Clinical Periodontology 35 (2008): 29-44.

14. Ishikawa I., et al. "Clinical application of erbium: YAG laser in periodontology". The International Academy of Periodontology 10.1 (2008): 22-30.

15. Dimofte A., et al. "The value of platelet rich fibrin in bone regeneration following tooth extraction". Romanian Journal of Oral Rehabilitation 9.3 (2017).

16. Izumi Y., et al. "Current and future periodontal tissue engineering”. Periodontology 200056.1 (2011): 166.

17. Aoki A., et al. "Periodontal and peri-implant wound healing following laser therapy". Periodontology 200068.1 (2015): 217269.

18. Aly L. "Piezoelectric surgery: Applications in oral and maxillofacial surgery". Future Dental Journal 4.2 (2018): 105-111.

19. Kim Y., et al. "Effect of low-level laser treatment after installation of dental titanium implant-immunohistochemical study of RANKL, RANK, OPG: An experimental study in rats". Lasers in Surgery and Medicine The American Society for Laser Medicine and Surgery 39.5 (2007): 441-450.

20. Shakouri S., et al. "Effect of low-level laser therapy on the fracture healing process". Lasers in Medical Science 25.1 (2010): 73-77.
21. Farivar S., et al. "Biological effects of low level laser therapy". Lasers in Medical Science 5.2 (2014): 58.

22. Dos Santos Santinoni C., et al. "Influence of low-level laser therapy on the healing of human bone maxillofacial defects: A systematic review". Journal of Photochemistry and Photobiology B: Biology 169 (2017): 83-89.

23. Kocyigit I., et al. "A comparison of the low-level laser versus low intensity pulsed ultrasound on new bone formed through distraction osteogenesis". Photobiomodulation, Photomedicine, and Laser Surgery 30.8 (2012): 438-443.

24. Whelan H., et al. "Effect of Light-emitting Diode Irradiation on Wound Healing.

25. Tamura M. "Non-invasive monitoring of the redox state of cytochrome oxidase in living tissue using near-infrared laser lights". Japanese Circulation Society 57.8 (1993): 817-824.

26. Beauvoit B., et al. "Correlation between the light scattering and the mitochondrial content of normal tissues and transplantable rodent tumors". Analytical Biochemistry 226.1 (1995): 167-174.

27. Mester A., et al. "Experimental imunological study with radiological application of low power laser: Laser in der Medizin Laser in Medicine". Springer (1998): 509-512.

28. Sommer A., et al. "Biostimulatory windows in low-intensity laser activation: lasers, scanners, and NASA's light-emitting diode array system". Journal of Clinical Laser Medicine and Surgery 19.1 (2001): 29-33.

29. Prakash S and Thakur A. "Platelet concentrates: past, present and future". Journal of Oral and Maxillofacial Surgery 10.1 (2011): 45-49.

30. Dohan D., et al. "Slow release of growth factors and thrombospondin-1 in Choukroun's platelet-rich fibrin (PRF): a gold standard to achieve for all surgical platelet concentrates technologies". Growth Factors 27.1 (2009): 63-69.

31. Yamashita Y., et al. "Stability of platelet-rich fibrin in vivo: histological study in rats". Journal of Oral Tissue Engineering 14.2 (2016): 83-90.

32. Kotsakis G., et al. "Extraction socket management utilizing platelet rich fibrin: a proof-of-principle study of the "Accelerated-early implant placement" concept". The Journal of Oral Implantology 42.2 (2016): 164-168. 
33. Tabrizi R., et al. "Does platelet-rich fibrin increase the stability of implants in the posterior of the maxilla? A split-mouth randomized clinical trial". International Journal of Oral and Maxillofacial Surgery 47.5 (2018): 672-675.

34. Mester E., et al. "Effect of laser rays on wound healing". The American Journal of Surgery 122.4 (1971): 532-535.

35. Khadra M., et al. "Low-level laser therapy stimulates bone--implant interaction: an experimental study in rabbits". Clinical Oral Implants Research 15.3 (2004): 325-332.

36. Khadra M., et al. "Laser therapy accelerates initial attachment and subsequent behaviour of human oral fibroblasts cultured on titanium implant material: A scanning electron microscopic and histomorphometric analysis". Clinical Oral Implants Research 16.2 (2005): 168-175.

37. Akimoto K., et al. "Evaluation of titanium implants placed into simulated extraction sockets: a study in dogs". The International Journal of Oral and Maxillofacial Implants 14.3 (1999): 351-360.

38. El-Talawy D., et al. "Radiographic evaluation of low intensity irradiation on immediately loaded implant supporting overdenture". Egyptian Den Journal 57.3 (2011): 2012-2018.

39. El-Kholey K and El-Shenaway H. "Role of diode laser in preservation of the marginal bone around early loaded endosseous implant". Life Science Journal 9.3 (2012): 940-943.

\section{Volume 5 Issue 10 october 2021}

(C) All rights are reserved by Abdel-Fattah M Amer.,

et al. 